\title{
BEAM BASED CHARACTERIZATION OF A NEW 7-POLE SUPERCONDUCTING WIGGLER AT CESR*
}

\author{
A. Temnykh ${ }^{\dagger}$, J. Crittenden, D. Rice and D. Rubin \\ Laboratory of Nuclear Studies \\ Cornell University, Ithaca NY 14953, USA
}

\begin{abstract}
The paper describes the beam based measurements of the magnetic field characteristics of the first 7-pole superconducting wiggler recently installed in CESR. The results are compared with the model prediction and with the estimates based on magnetic field measurements. It also presents results of the beam resonance mapping which was done by a $2 \mathrm{D}$ scan of betatron tunes while recording vertical beam size. The scan clearly exposed resonances excited by the wiggler nonlinear magnetic field components. In conclusion, the ways to optimize the wiggler magnetic field in order to reduce destructive effects on beam dynamics are discussed.
\end{abstract}

\section{INTRODUCTION}

The CESR low energy upgrade project calls for 12 superconducting wigglers installation to provide adequate radiation damping. The first 7-pole super-conducting wiggler [1] was built [2] and, after magnetic measurement [3], in the fall of 2002 was installed in CESR. A number of machine study periods were devoted to a beam based characterization of the wiggler magnetic field. Results of this characterization in comparison with the magnetic measurement and model prediction are described below.

\section{MODEL CALCULATION AND MAGNETIC MEASUREMENT RESULT}

The wiggler field integrals along straight lines and along wiggling beam trajectories are used for wiggler field characterization. Depending on the wiggler design, the difference between those integrals can be substantial. The straight line integrals calculated from model can be easily verified by a long flipping coil measurement. The measurement of the field integrals along beam trajectory can be done by using modified vibrating wire technique [6] or with a beam after wiggler installation in the ring.

Magnetic measurement results for $2.1 \mathrm{~T}$ and $1.9 \mathrm{~T}$ wiggler peak fields in comparison with a model calculation are presented in the Table 1. Moments $a_{n}$ and $b_{n}$ are the coefficients of the polynomial fit of the horizontal and vertical field integral dependence on horizontal position: $I_{x, y}(x)=\sum\left(a_{n}, b_{n}\right) \times x^{n}{ }^{1}$. Columns "str.line" and

\footnotetext{
${ }^{*}$ Work supported by National Since Foundation

† e-mail: abt6@cornell.edu

${ }^{1}$ In the case of straight line integrals the moments $a_{n}, b_{n}$ can be used
}

"str.coil" refer to calculated and measured straight line integrals. Columns labeled with "wgl.line" and "wgl.wire" are for integrals calculated and measured along wiggling trajectory of beam of $1.8 \mathrm{GeV}$ energy. The measurement technique is described in [3].

\begin{tabular}{|c|c|c|c|c|}
\hline \hline $\begin{array}{c}a_{n}, b_{n} \\
\frac{G m}{c m^{n}}\end{array}$ & \multicolumn{2}{|c|}{ Model } & \multicolumn{2}{c|}{ Magnetic measurement } \\
& str. & wgl. & str. & wgl. \\
& line & coil & wire \\
\hline \multicolumn{5}{|c|}{ Wiggler peak field $\sim 2.1 T$} \\
\hline$a_{1}$ & 0.0 & 0.0 & $1.53 \pm 0.01$ & N/A \\
$b_{1}$ & 0.0 & 1.33 & $-0.19 \pm 0.02$ & $2.5 \pm 0.15$ \\
$b_{2}$ & -0.29 & -0.28 & $-0.28 \pm 0.004$ & $-0.51 \pm 0.03$ \\
$b_{3}$ & 0.00 & -0.11 & $0.004 \pm 0.002$ & $-0.19 \pm 0.02$ \\
\hline \multicolumn{5}{|c|}{ Wiggler peak field $\sim 1.9 T$} \\
\hline$a_{1}$ & 0.0 & 0.0 & $1.37 \pm 0.01$ & N/A \\
$b_{1}$ & 0.0 & 0.83 & $-0.21 \pm 0.03$ & N/A \\
$b_{2}$ & -0.06 & -0.18 & $-0.02 \pm 0.001$ & N/A \\
$b_{3}$ & 0.01 & -0.10 & $0.007 \pm 0.003$ & N/A \\
\hline \hline
\end{tabular}

Table 1: Calculated and measured the wiggler integrated field characteristics.

Because of the model symmetry, the skew and normal quadrupole moments $a_{1}$ and $b_{1}$ of straight line integrals are equal to zero. However the straight coil magnetic measurement revels non-zero but negligible normal quadrupole moment $b_{1} \sim-0.2 \mathrm{Gm} / \mathrm{cm}$ and significant for beam dynamics skew quadrupole component $a_{1} \sim 1.57 \div 1.37 \mathrm{Gm} / \mathrm{cm}$. While the $b_{1}$ occurrence can be explained by a small error in pole geometry, the cause of relative large $a_{1}$ is not understood. The normal sextupole moment $b_{2}$ measured with straight coil is in good agreement with model calculation for both $2.1 \mathrm{~T}$ and $1.9 \mathrm{~T}$ wiggler fields. For $2.1 \mathrm{~T}$ it is $\sim-0.28 \mathrm{Gm} / \mathrm{cm}^{2}$ and for $1.9 \mathrm{~T}$ it is close to zero. The change in $b_{2}$ with a field level is due to specifics of the wiggler design. In the 7-pole design, the central pole is compensated by the two opposite polarity end poles. Because the magnetic field environment in the middle of the wiggler is different from the wiggler ends, the compensation can be provided in a limited range of excitation. Calculated and measured octupole moments $b_{3}$ are negligible.

Although the comparison between calculated and measured straight line integrals is the most convenient way

for integrated magnetic field representation in form $B_{y}+i B_{x}=\sum\left(b_{n}+\right.$ $\left.i a_{n}\right)(x+i y)^{n}$. In the case of integrals along wiggling beam trajectory this representation is not valid. 
to varify the model, the beam dynamics depends on the field integrals along wiggling beam trajectories. In [4] it was noticed that interference between the beam trajectory wiggles and field variation across the single pole, $B_{y}(x)$, results in an additional integrated field: $\Delta I_{y}(x) \simeq$ $-\frac{1}{2} L x_{p} \frac{d B_{y}(x)}{d x}$. Where $x_{p}$ and $L$ are the wiggling amplitude and the wiggler length. As $B_{y}(x)$ is a symmetric function, $d B_{y}(x) / d x$ and $\Delta I_{y}(x)$ are odd function of $x$. The latter generates the odd order moments (normal quadrupole $b_{1}$, normal octupole $b_{3}$ ) seen in column "wig.line" of the table 1. Magnetic measurement with a vibrating wire (column "wig.wire") confirmed existence of these moments but gave approximately two times bigger amplitudes. This inconsistency is likely to be a result of a not accurate calibration of the used vibrating wire technique.

\section{BEAM BASED CHARACTERIZATION}

\section{Wiggler generated coupling.}

Beam based measurement of the local coupling around the ring indicated $\sim 2 \mathrm{Gm} / \mathrm{cm}$ skew quadrupole moment generated by the wiggler, which is in good agreement with a magnetic measurement result. This component was compensated with skew quadrupole magnet installed near the wiggler.

\section{Wiggler generated betatron tune variation}

Vertical and horizontal betatron tunes were measured as a function of horizontal beam position in the wiggler at several wiggler field levels. For the beam displacement a closed orbit bump was used. The result of the measurement at $2.1 \mathrm{~T}$ and $1.9 \mathrm{~T}$ fields in comparison with tune variation obtained from the model tracking are plotted in Figures 1 and 2. In all cases one can see a reasonable consistency between calculation and measurement. The sextupole and

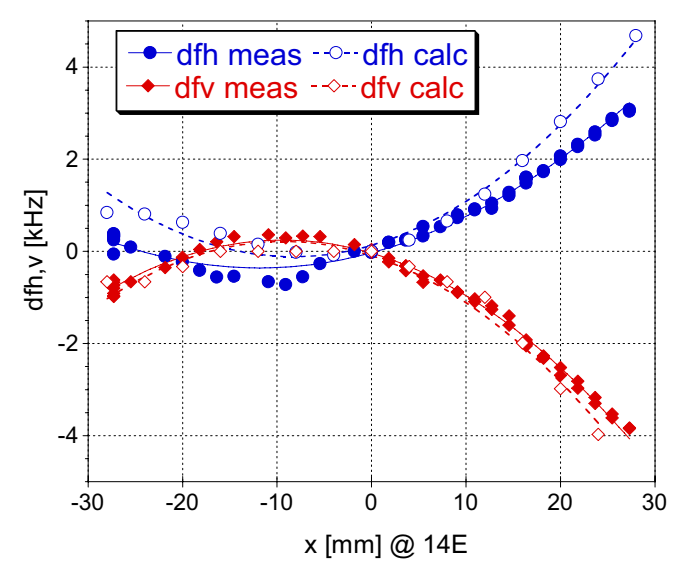

Figure 1: Measured and calculated betatron tune variation versus horizontal beam position in the wiggler at $2.1 T$ wiggler peak field.

octupole moments calculated from the coefficients of the

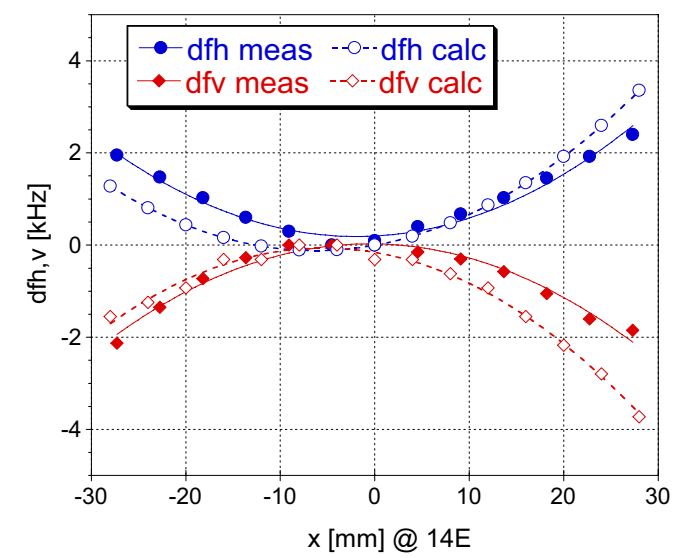

Figure 2: Measured and calculated betatron tune variation versus horizontal beam position in the wiggler at $1.9 T$ wiggler peak field.

polynomial fit of the measured horizontal tune variation are given in table 2. They are in good agreement with calculated, see column "wgl.line" in Table 1.

\begin{tabular}{|c|c|c|}
\hline \hline Moment & $2.1 T$ & $1.9 T$ \\
\hline$b_{2}\left[\mathrm{Gm} / \mathrm{cm}^{2}\right]$ & $-0.29 \pm 0.01$ & $-0.059 \pm 0.011$ \\
$b_{3}\left[\mathrm{Gm} / \mathrm{cm}^{3}\right]$ & $-0.082 \pm 0.002$ & $-0.10 \pm 0.004$ \\
\hline \hline
\end{tabular}

Table 2: The moments calculated from the measured dependence of horizontal tune on horizontal beam position in the wiggler.

\section{Nonlinear resonances excitation and tune plane appearance.}

The machine performance (luminosity, injection efficiency, beam life time and etc.) often critically depends on the appearance of the betatron tune plane. The tune scan, a measurement of beam characteristics as a function of the betatron tunes, exposing the tune plane resonance structure facilitates the choice of the working point and the structure analysis may help to reveal the cause of magnetic field nonlinearities affecting machine performance. To explore effect of the wiggler magnetic field on the nonlinear beam dynamics we made a series of tune scans with a vertical beam size measurement. Two examples are shown in Figures 3 and 4 . Here is depicted the vertical beam size as a function of betatron tunes on a $40 \times 40$ grid measured with zero and $2.1 T$ wiggler peak field and a flattened orbit. Both plots have the same vertical beam size scale. Vertical beam size was measured using synchrotron light monitor. To help identify resonances Figure 5 shows a resonance map corresponding to the experimental condition. On the map, only resonances seen in measurement are shown. The effect of the wiggler field on the beam dynamics can by clear observed by comparison of these two measurements. With wiggler field turned off, the scanned area 


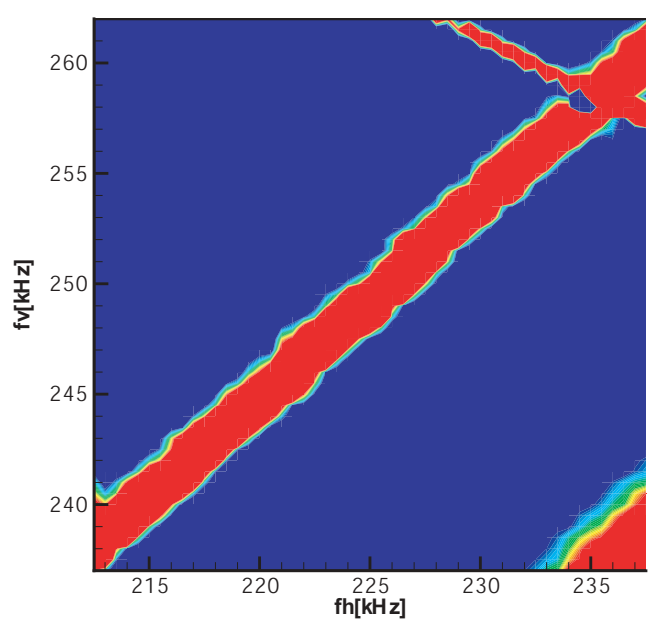

Figure 3: Vertical beam size versus betatron tune measured with CESRC-c wiggler turned OFF.

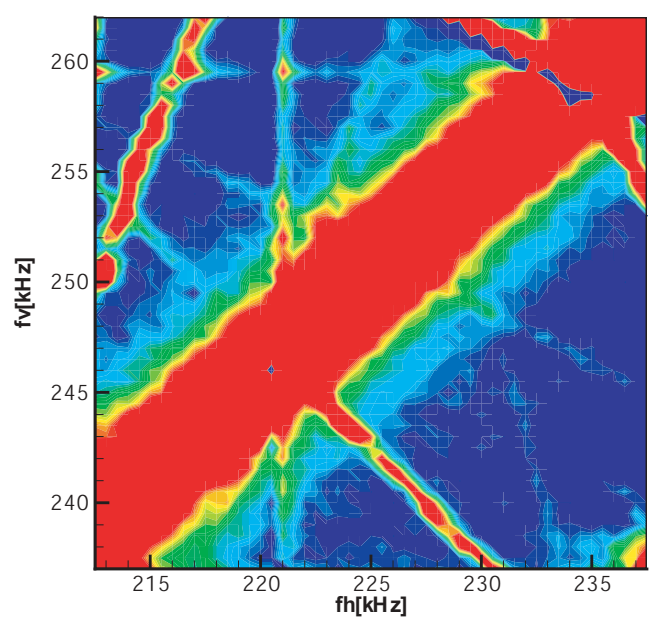

Figure 4: Vertical beam size as a function of betatron tunes measured at $2.1 \mathrm{~T}$ wiggler magnetic field.

is relatively clean. There are only 3 resonance lines: $-\mathrm{fh}+\mathrm{fv}$ $=0,-\mathrm{fh}+\mathrm{fh}-\mathrm{fs}=0, \mathrm{fh}+2 \mathrm{fv}+\mathrm{fs}=2 \mathrm{f} 0$. With $2.1 T$ wiggler field one can see 8 additional "working" resonances: $-3 \mathrm{fh}+\mathrm{fv}=-$ $\mathrm{f} 0, \mathrm{fh}+\mathrm{fv}-3 \mathrm{fs}=\mathrm{f} 0,3 \mathrm{fv}=2 \mathrm{f} 0, \mathrm{fh}+2 \mathrm{fv}+2 \mathrm{fs}=2 \mathrm{fO}, 4 \mathrm{fh}+\mathrm{fv}=3 \mathrm{f} 0$, $2 \mathrm{fh}+\mathrm{fv}+2 \mathrm{fs}=2 \mathrm{f} 0,2 \mathrm{fh}-2 \mathrm{fs}=\mathrm{f0}$ and $-3 \mathrm{fh}+\mathrm{fv}+\mathrm{fs}=-\mathrm{f} 0$ covering much bigger area then the previous. Based on this observation one can conclude that in the given case the wiggler nonlinearity is a major player in a nonlinear beam dynamics.

A tracking simulation of the observed effect of the wiggler field on beam dynamics is progress.

\section{CONCLUSION}

The magnetic field of the first 7-pole super-conducting wiggler was characterized by the magnetic and beam based measurements. Reasonable agreement has been found between these measurements and the model calculations. Results of tune plane scanning suggest that the effect of the

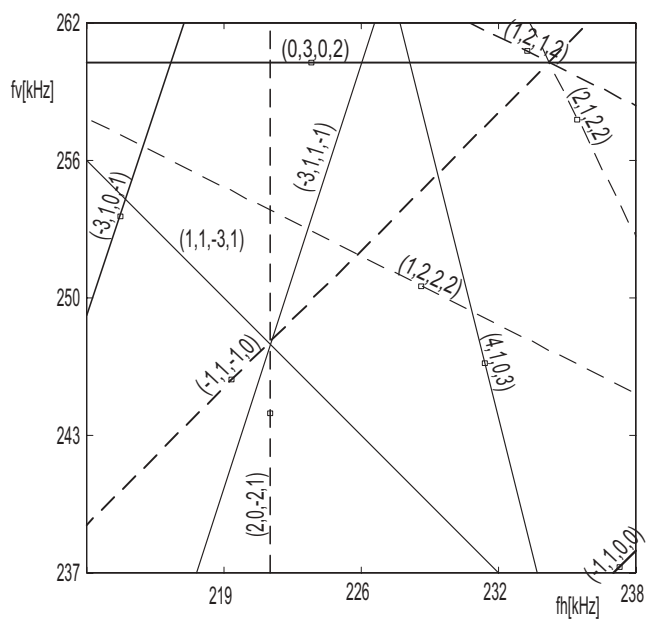

Figure 5: Resonance map for a scanned tune plane area. Shown are resonances seen in measurement. Labels $(p, q, r, n)$ indicate $p f_{h}+q f_{v}+r f_{s}=n f_{0}$ resonance lines.

wiggler field nonlinearity on beam dynamics dominates over the effect from the rest of the ring and could compromise machine perfomance.

The part of the wiggler nonlinearity problem can be associated with the design specific. In symmetric 7-pole configuration the central pole is compensated by two opposite polarity end poles. But because of very different magnetic environment at the ends and in the middle of the magnet this compensation can not be fulfilled completely. The corroboration of the modeling of the CESR-c wigglers by the measurements presented above was an important step in the decision for the final 8-pole configuration of the wigglers. In 8-pole design each pole is compensated by the identical pole of opposite polarity. This provides better field nonlinearity compensation in a wider range of the wiggler field excitation.

\section{REFERENCES}

[1] James Crittenden et al., Design Considerations for CESR-c Wiggler Magnets, to be published in PAC2003 proceedings.

[2] David Rice, Richard Gallagher et al., Production and Testing Considerations for CESR-c Wiggler Magnets, to be published in PAC2003 proceedings.

[3] A. Temnykh, Vibrating Wire and Long Integrating Coil Based Magnetic Measurements of a 7-pole SuperConducting Wiggler for CESR., to be published in PAC2003 proceedings.

[4] J. Safranek et al., Nonlinear Dynamics in SPEAR Wigglers, EPAC' 2000, p.295

[5] This measurement technique was first developed by the ID group at ESRF. See Developpment de Banc de measures magnetiques pour undulateurs et wigglers, D. Frachon Thesis, April 1992.

[6] A. Temnykh, Some aspects of the use of Vibrating Wire Technique for a wiggler magnetic field measurement, Preprint CBN 01-17, Cornell 2001. 